\title{
Dear recommender,
}

We are pleased to send you a revised version of our manuscript.

We would like to thank the reviewers and you for their time devoted to the review of the manuscript, for the encouraging comments and for their suggestions, which I have no doubt, have enabled us to improve the manuscript.

We have taken all of your feedback into account in the attached revised manuscript, and Supplementary materials. We would like to emphasize that following the suggestion by reviewer 1 we added the test of the artificial reef shape which led us to slightly modify the title of the manuscript in order to better reflect our findings:

Artificial reefs geographical location matters more than shape, age and depth for sessile invertebrate colonization in the Gulf of Lion (NorthWestern Mediterranean Sea)

We also provide a letter for each reviewer addressing their respective comments in details.

We sincerely thank you for your time and effort that goes into the publication of this paper.

Best regards

Sylvain Blouet, Katell Guizien and Lorenzo Bramanti

\section{Reviewer 1}

\section{Comments to the Author:}

The research article written by S. Blouet, L. Bramanti and K. Guizien builds an important knowledge for ecosystem management related to artificial reef deployment. Their work emphasizes the idea that artificial unit geographical position matters more than its immersion duration or depth. Their results suggest that implementation of such artificial units should not be done randomly but instead by selecting areas which could maximize recruitment and settlement of benthic organisms.

The article is well written, short and gets right to the point! I could still possibly see a few critical issues or some room for improvements. I will (try to) explain what are the critical issues I refer to and then detail what could eventually be improved along the manuscript.

\section{Critical comments:}

\#1 I'm not a statistical expert but it seems to me that the artificial reefs investigated here present a vast array of shape and size. I'm particularly concerned that these different designs could have a profound effect on the recruitment and the settlement of benthic organisms to start with. The figure 4 and the supplementary file are reinforcing this idea that the shape of the artificial unit could strongly influence your analyses.

So, is there any site where you could eventually test this assumption or eventually any references you could use to cover this aspect?

Your suggestion is totally relevant. The difficulty was that as we did not control the artificial reef deployment that occurred in the past, it was not evident how to test for the influence of the shape 
without interaction with other factors. The only site where the shape factor could be tested statistically (with replicates) was VLR (VLR1\&VLR2) with ARs of different shape but of the same age and at depths less than $20 \mathrm{~m}$. In GRU, we re-organized the grouping of sampling units into three sites, enabling us to separate ARs with different shapes in the same location (GRU1 and GRU2) and ARs with the same shape in different locations (GRU2 and GRU3). However, due to the lack of a replicated sampling unit for the shape "heaps of telegraph poles", we could not test statistically the difference between these three sites, but only commented on them. The manuscript was revised to incorporate the shape test in the Title, Abstract (L. 18-23), Key words, Material and Methods section (L.94; 101-102; 105; 106; 184-189; 198-200), in the Results section (L225-233) and in the Discussion (315-324).

\#2 Can you precise if the analyses were done on abundance data (L120-121) or on presence/absence data (L180). I am quite confused about how the data were processed but I admit I could have misunderstood this since your material and methods refers to presence/absence data but your results are not referring to it anymore.

The analyses were performed on presence/absence data only.

For clarification, we have reformulated the L184 in the statistical analysis paragraph of the Material and methods section. In the results section of the manuscript, we described the presence of a species by using the verb "detected".

\#3 I understand that the use of different structure designs, at different time and different depths make your statistical design unbalanced and not all comparisons could be made (L183-188).

However, this choice of format will probably make things difficult for the reader I would suggest to clearly state what are the factors that you want to test and summarize which group of ARs was used for testing each of those factors.

A suggestion here (just a suggestion) would be to extend your supplementary information by adding information about grouping and statistical tests. The reader will be able to see from this table which unit, into groups have been used to test each assumption. What do you think?

According to your suggestion, we have included the name of the sampling units used for testing the different factors in the text (L. 102-103; 105; 112; 191-192; ) but also in fig 3 to locate them in the study area. We also added a table (table 2: supplementary material) summarizing the sampling units and factor testing layout for each statistical analysis. .

\#4 It seems to me that you are trying to get rid of the structural complexity here as a factor affecting the recruitment and the settlement of benthic organisms. I would suggest 1-try to test structure it if you have different structures with the same age immersed at the same site; 2-provide some kind of trend that you observe in your data which could minimize the structure effect (you have similar benthic composition found on different structures with the same age); or 3-acknowledge that structure could eventually affect your results but that it could not be tested in this present study. Please refer to answer to comment \#1

I will detail hereafter some minor issues with the manuscript and suggest some alternatives to help the reading.

Abstract:

\#5 At first, I kind of feel like the research question was missing from your abstract. I thought that it could be improved by introducing what is missing in the literature and what your study is dealing with: "While most studies focused on short term colonization history, we proposed to test immersion duration, geographic location and depth on the colonization of artificial reefs."

After several readings I'm not too sure about it anymore, so feel free to consider it as a suggestion only. 
\#6 L15. Five sessile species [...].

Correction applied. L16

\section{Introduction:}

\#7 L35: Suggestion only => "Beneficial effects such as increases in fish biomass [...]." Correction applied. L16

\#8 L39: When I read "The fish production argument [...]" I am sort of expecting to also read "The fish concentration argument [...]".

We reworded the previous sentence in L38-39

\#9 L62-76: I think after such a nice piece about the effect of the structural complexity you cannot escape from investigating this parameter in this present study (see critical comments).

See answer to comment \#1 on the revision of the manuscript taking into account the complexity.

\#10 L77: I would mention in this sentence what is long term and remove the last sentence of the introduction which totally belong to the M\&Ms section.

Correction applied.

We have integrated the sampling spatial scales into the abstract to strengthen the spatial analysis of our study in L. 19-21.

\#11 L81: Golf de Lion (GDL) but Gulf of Lion (GoL)? If this is already used in the literature, I would suggest to follow otherwise feel free to ignore this comment.

Correction applied throughout I the entire manuscript

\section{Material and Methods:}

\#12 L97-98: Is there a reason for removing the artificial reefs deployed in 1992-1999?

The ARs of intermediate age (1992-1999) were deployed in only in two sectors (AGM and AGD) which means that the test of the factor age would not have been more powerful using them than using the oldest ones, that were deployed in two sectors as well (CST and AGD). Moreover, their position was not precisely known and given their small size (a few meters), finding them was difficult. We therefore decided to focus on the oldest and youngest ARs of the Gulf of Lion, maximising the age difference between ARs. We clarified this choice in the Material and Methods section on L.94.

\#13 L101: I know that this information is already in your supplementary but I would give to the reader the full name of those sites, even if there are in French. I believe your research paper will attract a large panel of reader, not restricted to researchers. A clear communication on your M\&Ms section will really increase the visibility of your paper.

We thank you for the advice and added the full names in M\&M and on Figure 1.

\#14 L103: So, each geographical sector has been divided into 2 or 5 sites. Into each of those sites, 3 sampling units were set up by pooling ARs to reach a minimum surface of $306 \mathrm{~m} 2$ par site. Is that correct?

It is correct.

I am a little bit concern here since your figure 1 is not showing this so I think the reader will easily get lost. 
Similarly, to my previous critical comment I would clearly explain the grouping and subgrouping and summarize this information in your supplementary table (or eventually into another small table). For instance, I would suggest to name these groups (Groupe I, or group "alpha", or group "immersion duration" to refer to what was tested, directly). Then I would use this information in the M\&Ms / Statistical analysis ("To test for XXX, we used group XXX which consist in XXX (See Supplementary file)". I think this will be a lot easier for the reader.

In accordance with your suggestion, we have included more precisely the sites names in the text but also in fig 3 to locate them in the study area. Moreover, we added a table (table 2: supplementary material) summarizing the analyses layout.

\#15 L113: Just to make sure, sampling units are artificial reefs, right? Also, according I would follow the nomenclature of your supplementary and use GRU2 (or GRU-2) to mention the second sampling unit of GRU.

Sampling units can include one or multiple neighboring ARs in order to reach a surface of a minimum of $89 \mathrm{~m} 2$ as stated in L.108-111.

As suggested, we corrected the name of the sites in the sector GRU and refer to GRU1, GRU2 and GRU3 throughout the text (L.111-113).

\#16 L120-123: Did you count all individuals directly underwater or did you take photographs of each ARs and analyze the photographs later?

The inventories were carried out in scuba diving with a direct visual count. This information was added on L. 124-125.

How did you account for individuals from very small size? In my field (scleractinian corals), we usually always precise that all individuals larger than $5 \mathrm{~cm}$ have been accounted in the survey because divers will always have missed newly recruited organisms. Maybe you could have this kind of information and detail a little more how specimens were accounted for.

We have revised L. 126, adding the minimal size for the detection of individuals. We estimate that below $2 \mathrm{~cm}$ it could have been difficult to distinguish between species of the same family.

\#17 L121: "44 sampling units" could also be a little confusing here. I think a new format of Supplementary file could also help here.

The sentence has been changed according to the suggestion. The reference to " 44 sampling units" and " 80 ARs" was eliminated to avoid confusion and replaced by a reference to the total surface inventoried L.125. We think that the text is now clearer and hence it is no more necessary to change the format of the supplementary material.

\#18 L124-176: I have several problems here. First of all, despite the fact that we are in the "Colonization assessment" section, this is more related to species distribution and traits. In addition, even if it's important to explain why you chose these 5 species (and your choice is mainly based on their abundance across their distribution range), I feel like a lot of this should be move into the Discussion section. Finally, even if all those information are critical for your study, a table summarizing species traits would be welcome here.

So as a suggestion, I would create another sub-section after "Study area and stratified sampling design" to refer to why you pick those 5 species. This section could explain the distribution of these 5 species and briefly mentioned their life history traits with reference to a recapitulative table. Then the "Colonization assessment" will follow explaining how you collect your data. To me, most of the information from L147 to L176 should be discussed (and this information is indeed, discussed in your manuscript, which makes it redundant). 
In accordance with your suggestions, we have added "species selection" in the paragraph title, to better reflect the message of the paragraph in L. 123.

We also added in table 1 additional information from this paragraph such as "spawning period, fecundity, life expectancy, age at sexual maturity, larval type, planktonic larval duration (PLD), abundance in the GoL" to summarize the information required and help the understanding of the reasoning presented in the discussion.

We believe that the section describing how we retrieved the life traits of species should be kept in the M\&M because it effectively guided the species selection prior to our survey and we think that transferring this paragraph in the discussion would not help readability.

\section{Results:}

No particular comments on the results section.

\section{Discussion:}

\#19 L245-247: The first sentence of the discussion is way to "heavy". Also, I would explain what you mean by "effective integration". We have revised the beginning of the discussion (L. 269-273) to better explicit the notion of "effective integration".

Then would you say that the efficiency of the ARs for recruitment and colonization is essential to assess? Or just the dynamic of colonization?

Actually, the efficiency of ARs should be defined with respect to an objective. If the objective was supporting hard bottom diversity by increasing the surface for hard bottom species, ARs colonization assessments should be the first step, if one wants to rate their integration into a network of natural habitats. Then for a successful integration it is necessary to understand if there are also exchanges of AR populations towards the natural habitat (not studied here).

\#20 L247-249: I would tend to disagree with this sentence. The ARs there have been colonized by a greater number of species for sure, probably because ARs provide a suitable hard substrate for them. Your study "demonstrate" way more than just this simple observation and it arrives later from L249251.

We agree and reformulated L. 273-275 accordingly.

\#21 L291-296: https://onlinelibrary.wiley.com/doi/full/10.1002/ece3.6047 or https://www.sciencedirect.com/science/article/pii/S0141113605000590.

I'm not an author of these references but it seems to me that reef complexity can affect colonization and effective recruitment.

From my point of view this question is a little bit missing in this study. I understand the limitations you have here but I would suggest to either 1-try to test structure it if you have different structures with the same age immersed at the same site; 2-provide some kind of trend that you observe in your data which could minimize the structure effect (you have similar benthic composition found on different structures with the same age); or 3-acknowledge that structure could eventually affect your results but that it could not be tested in this present study.

With your help we have therefore integrated the shape into the factors tested on colonization and revised this part of the discussion. Nevertheless, we considered the shape and not the complexity because we believe that the calculation of the complexity is a difficult exercise, as it includes different metrics (e.g. rugosity, TRI, roughness, fractal dimension). Moreover, we also consider that in studies evaluating colonization as a function of complexity, the surface should be controlled, which is the case in our study and was not done in by Rouse et al., (2019) and Perkol-finkel et al., (2016). In these studies, complexity and surface were confounded as we argue in L313-321.

Finally, our approach by considering the presence/absence of species also limits the bias linked to the area. 


\section{Figures and tables:}

Fig3: The color for artificial reef area prospected and Leptogorgia is almost the same.

Correction applied. L16

\section{I would love to have a few more comments directly to the authors which they could explore further only if they think it fits their manuscript:}

After reading $L 45-48$ and $L 275$, I feel like the message could be: If the location is ideal based on larvae availability and current (which maximize the availability of larvae in an area) would you recommend to set up ARs on sandy substrates? And how does this will affect sandy ecosystems (because even if they are less productive, they are still important and unique ecosystems with their own biodiversity and function)?

We do not recommend the deployment of ARs on sandy environments as indeed, there is a cost to the perturbation of sandy ecosystems as well. However, if the decision to deploy ARs is made to support fisheries or hard bottom diversity resilience, our findings indicate the location in the region should be taken into account prior to other local factors to guide the deployment and evaluate the benefit and risk of the deployment.

Also, in this study you targeted 5 species with contrasted life history trait but how would you connect your study with other species sharing (or not sharing) the same life history traits. Would you make some assumptions in your manuscript to deal with those cases?

At this level, it is difficult to extend our results on these 5 target species to other species, because not all the possible life history traits combinations have been taken into account in our study (high fertility, low PLD and high abundance or high fertility, high PLD and high abundance.

Moreover, life history traits of most benthic invertebrates are poorly known, as their distribution in the natural habitat, making the extension of our results difficult. Nevertheless, we are working on a more precise mapping of the abundance of hard-bottom benthic invertebrates in the Golf du Lion.

An interesting assumption here in your manuscript is that ARs might not have reach a stable state yet (L270-273) due to the presence of S. spallanzanii. However, you also mentioned that 1- this species is relatively rare in natural settings and abundant on artificial structures. In this case would you eventually consider that these particular artificial reefs cannot mimic natural hard substrates? Or that the natural succession cannot occur on these artificial units?

Depending on their location, ARs can mimic the natural environment, which was the case for ARs on AGD, where the 5 species were detected. Thus, successions equivalent to the natural environment can occur, however the presence of $S$. spallanzanii suggests that these environments are subjected to excessive disturbance to reach a maturity comparable to the environment. In summary, the succession can be equivalent to the natural environment but the maturity of the AR was not equivalent to the natural environment.

\section{Reviewer 2}

\section{Comments to the Author:}

Dear Sylvain Blouet, Lorenzo Bramanti, and Katell Guizien, 
I enjoyed reading your manuscript. Long term monitoring data acquired during these projects are of extremely importance to managers since they can provide crucial ecological patterns not discerned from short term studies.

The authors do an overall great job at introducing the topic of artificial reefs (ARs) and their importance. Perhaps an additional sentence may be added regarding additional negative effects (e.g., chemical pollution, marine debris, beacons for fishermen).

We thank you for the suggestion but we feel it is out of the scope of our paper to discuss general negative effects linked to artificial reefs. However, specific negative effects in relation to the colonization of ARs by benthic invertebrates have been mentioned in the discussion section (L.370372).

I was able to follow the materials/methods sections, as well as the results and discussion. This is perhaps, along with the data provided, their strongest quality of their written work. I also really enjoyed their figures and tables because they were easy to follow and read.

In my opinion, their conclusion is their weakness, and feel the authors should dedicate additional time to this section. The authors mention since the introduction that ARs are primarilay deployed to reduce fishing pressues in certain fisheries, if not all (non acessable scuba sites may be protected from these). However, the authors do not provide data regarding the biomass, abundance, and species diversity of fish associated with the deployed ARs. They only provide data on five sessile invertebrates which dominate these ARs. I only mention this because of how the manuscript is ended, "The present study advocates accounting for the geographical arrangement in planning ARs deployment to enhance fish productivity while avoiding the spread of invasive species.", but the authors do not provide evidence of such.

Your remark is relevant. We reformulated our conclusion, which was speculative as we do not have data on the colonization of the ARs by fishes. We have therefore rewritten the last sentence to stick to our results regarding the hard substrate habitat extension for benthic invertebrates.

I wonder why the authors decided to just analyze the dataset from the years 1985 and 2000 (on forward)? The dataset from 1992-1999 may provide additional and important data pertaining to the area. These may allow the authors to observe additinal patterns which may strengten their scientific argument.

The ARs of intermediate age (deployed in of 1992-1999) were located only in two sectors (AGM and AGD) which means factor age testing would not have been more powerful using them than using the oldest ones, that were deployed in two sectors as well (CST and AGD). Moreover, their position is not precisely known and given their small size, finding them was difficult. We therefore decided to focus on the oldest and youngest ARs of the Gulf of Lion, maximizing the age difference between ARs. We clarified this choice in the Material and Methods section on L.94.

Lastly, I would like to congratulate the authors for their effort and dedication. Best regards,

\# L3. Inventoried... recorded?

Correction applied.

\# L17 Please state for how long did the ARs that were immersed in 1985 lasted underwater for. Please state the exact depths they were deployed at.

Actually, the ARs deployed in 1985 are still underwater. This allowed to perform our study on old ARs (deployed in 1985, more than 35 years) and young ARs (deployed in the 2000s, more than 10 years). 
We have now integrated the depth ranges where the ARs were deployed (L. 19). Exact depth can be found in Supplementary Material Table 1.

\# L31 they have also served as easier spots to capture/kill the aggregating fish. Perhaps this should also be mentioned.

The attractor effect of ARs which facilitates captures was mentioned originally in L35-38 and have been now reformulated according to the comments of reviewer \#1 in L. 35-37 as follows: "Beneficial effects such as increase in fish biomass and capture efficiency near ARs have been reported (reviewed by Bohnsack and Sutherland, 1985; Tessier et al., 2014) but led to a debate opposing attraction vs production regarding the effects of ARs on fishery (Grossman et al., 1997)".

\# L44 is this word suppose to be here? Seems out of place.

Correction applied

\# L66 suggestion: "to mimic"

Suggestion applied

\# L188 Please include, in parenthesis, your ID number, thank you very much .

Although we are not sure to understand this comment correctly, , we have added a Table 2 in the supplementary material which summarizes the statistical tests layout, indicating factors and the sampling units used to test for the factors.

\# L196 Please include references of figures if conducted

We now added a reference to the supplementary material Table 2 which summarizes the univariate analyses layout in the M\&M (L.197) and refer to tables 5 and 7 in the Results (L.251; 264).

\# L196 Please include reference to figure \#\#

The results corresponding to post hoc tests mentioned in this line of the M\&M are described in L.251-254, and Table 3 in the supplementary material has been added.

\# L196 please include supporting reference

Reference was added.

\# L357 Please italicize all scientific names

Correction applied. 